\title{
Optimal Control of a Surface Vehicle to Improve Underwater Vehicle Network Connectivity
}

\author{
Camila C. Françolin* and Anil V. Rao \\ University of Florida, Gainesville, Florida 32611 \\ and \\ Christiane Duarte $\stackrel{\ddagger}{\ddagger}$ and Gerald Martel $\underline{\underline{s}}$ \\ U.S. Naval Undersea Warfare Center, Newport, Rhode Island 02841
}

DOI: $10.2514 / 1.1010002$

\begin{abstract}
The use of an autonomous surface vehicle as an auxiliary agent to improve connectivity in a network of autonomous underwater vehicles is investigated. An algorithm is developed that consists of an optimal waypoint generator and a minimum-time guidance law that is used to steer the vehicle to the waypoint. This algorithm is used together with a communication architecture to improve underwater vehicle connectivity in a region of interest. The approach is simulated using various underwater vehicle configurations, both with and without the autonomous surface vehicle, where it is found that network connectivity is improved significantly via the inclusion of the autonomous surface vehicle. An in-water hardware test is then performed and is shown to be consistent with the simulation results. The results of this study show that adding an autonomous surface vehicle to an underwater vehicle network can improve the connectivity of the network.
\end{abstract}

\section{Introduction}

A N IMPORTANT problem in cooperative control of multiple-agent air, ground, and underwater vehicle systems is maximizing the connectivity of the mobile sensor network [1-6]. Several different definitions of network connectivity have been previously proposed. Global message or worst-case connectivity is used in systems where it is important to keep all of the nodes in a network connected [7]. In the case of global messaging, the concept of a minimal spanning tree provides a measure of the connectivity of the network [7-9]. Network bisection connectivity [7] is based on the eigenvalues of the graph's modified Laplacian matrix and comes from the minimum cut tree problem [10]. Finally, $k$ connectivity is used in situations where it is desired to guarantee connectivity in the event that some subsets of the network are disabled [8,9]

In the case of underwater vehicles, applications of cooperative control where network connectivity is important include ocean floor mapping, mine sweeping, and area patrolling. Such tasks are usually accomplished through coordinated control of a network of vehicles, making it necessary to incorporate communication and connectivity constraints to the motion of each vehicle in the network. Previous research on this topic has focused on guaranteeing global network connectivity by restricting the motion of the underwater vehicles so that each vehicle is required to stay within communication range of one another [1-6]. Reference [3] relied on maintaining vehicle distances and line-of-sight restrictions, while [5] devised a plan to improve connectivity in a network by a method of reactive behavior coupled with planning. Reference [6] devised a reactive control strategy in which the controller is a weighted sum of two goals: the first term guides each robot to its goal position, and the second term maintains the constraints that need to be satisfied to maintain network connectivity. While these previously developed algorithms have been successful in keeping the network globally connected, requiring the vehicles to be within a specified separation distance of one another can often prove to be restrictive because the vehicles then must choose between accomplishing the mission and maintaining connectivity [4].

In this paper, we consider a decentralized network of autonomous underwater vehicles (AUVs) and we explore the possibility of improving network connectivity via the inclusion of an autonomous surface vehicle (ASV) to the network, where the ASV relays status messages between AUVs that might otherwise be disconnected. We propose a controller for the ASV that seeks to maximize the number of possible connections in the network of AUVs. Thus, the ASV acts as a bridge that relays information to the vehicles in the network. Because the motion of the underwater agents is unrestricted, it is not possible to guarantee global connectivity of the network. Instead, in this research, we seek to locally improve the connectivity of a subgroup of agents in the network. To ensure that no part of the network is disconnected for extended periods of time, we develop a behavioral outer-loop controller for the ASV. This outer-loop controller decides at the start of each outer-loop control cycle whether to choose the solution of a continuous-time optimal control problem or to position the ASV in a neighborhood of a vehicle that has been outside of communication range for an extended period of time: a polling behavior.

Improvements in the network connectivity were quantified using two metrics referred to as the connectivity number $N_{C}$ and the propagation number $N_{P} . N_{C}$ and $N_{P}$ provide measures of the number of direct and indirect connections, respectively, in the network. Previous research on network connectivity has often employed different metrics from those that we propose in this research. Such metrics include the algebraic connectivity of the network, denoted as $\lambda_{2}$, which is the second smallest eigenvalue of the network Laplacian. Variable $\lambda_{2}$ is a measure of the global connectivity of the network [6,11]. Specifically, a nonzero value of $\lambda_{2}$ implies that the network is globally connected [12]. In this research, we are not interested in a measure of the global connectivity of the network but are instead interested in the average number of connections (or average valency) in the network as a measure of local improvement in network connectivity.

Presented as Paper 2010-7572 at the 2010 AIAA Guidance, Navigation, and Control Conference, Toronto, 2-5 August 2010; received 4 July 2011 ; revision received 8 January 2012; accepted for publication 18 January 2012. Copyright $\odot 2012$ by Camila Clemente Françolin and Anil Vithala Rao. Published by the American Institute of Aeronautics and Astronautics, Inc., with permission. Copies of this paper may be made for personal or internal use, on condition that the copier pay the $\$ 10.00$ per-copy fee to the Copyright Clearance Center, Inc., 222 Rosewood Drive, Danvers, MA 01923 ; include the code and $\$ 10.00$ in correspondence with the CCC.

*Ph.D. Student, Department of Mechanical and Aerospace Engineering; francoli@ufl.edu.

${ }^{\dagger}$ Assistant Professor, Department of Mechanical and Aerospace Engineering; anilvrao@ufl.edu. Associate Fellow AIAA (Corresponding Author).

‡Computer Scientist, Autonomous and Defensive Systems Department; christiane.duarte@navy.mil.

${ }^{\S}$ Computer Scientist, Autonomous and Defensive Systems Department; gerald.martel@navy.mil. 
The contributions of this paper are as follows: 1) a new concept to improve local network connectivity through the inclusion of an additional bridge agent without having to alter the motion of the agents in the network, 2) testing of the guidance law in simulation, and 3) a hardware implementation of the approach in an in-water test.

This paper is organized as follows. In Sec. II, we provide an overview of the network connectivity problem of interest in this research. In Sec. III, we describe the metrics that are used to assess the connectivity of the underwater vehicle network. In Sec. IV, we provide details on the algorithm used for guidance and control of the ASV. In Sec. VI, we show simulation results for different configurations of the AUV network. In Sec. VII, we show the results of our approach that were obtained from an in-water hardware test. In Sec. VIII, we provide a discussion of the approach. Finally, in Sec. IX, we provide conclusions.

\section{Overview of Motivating Problem}

Consider the problem of maintaining communication in a decentralized network of $n$ AUVs that move in a region of interest in a body of water. The position of the $i$ th vehicle in the network $A_{i}(i \in 1, \ldots, n)$ in three-dimensional Euclidean space $\mathbb{E}^{3}$ at time $t$ is denoted $\mathbf{p}_{i}(t)=$ $\left(x_{i}(t), y_{i}(t), z_{i}(t)\right)(i=1, \ldots, n)$. The network of AUVs is dynamic in size and is reconfigurable; that is, any agent in the network can change its pattern of movement or can join or leave the network based on changing mission requirements. Furthermore, each AUV has no restriction on its motion based on network connectivity requirements. The AUVs communicate with one another through a status message, where the status message includes both information about the location and velocity of that particular agent and information about the awareness that a particular agent has about the overall state of the network. The status messages that are broadcast throughout the network are essential in order to successfully complete the mission because the behaviors built into each AUV use the information contained in these status messages to make mission-level decisions.

In the mission of interest in this paper, each underwater vehicle $A_{i}(i=1, \ldots, n)$ patrols a specified zone and moves in a fixed pattern through a series of predetermined waypoints that are confined to that zone. The region to be patrolled is divided into concentric rings (or tiers) that encircle a region of interest. Each vehicle in the network communicates through an underwater acoustic modem, where each modem transmits its status message sequentially. The use of sequential transmission prevents an overrun in communication, ensuring every communication is heard throughout the network. Each AUV broadcasts its message once before reaching and after leaving a waypoint, thus creating a pattern that discretizes the real-time mission into communication cycles of length $T_{c}$. Specifically, at a particular time $t$, each agent $A_{i}(i \in 1, \ldots, n)$ broadcasts to the network its estimated position $\mathbf{p}_{i}(t)=\left(x_{i}(t), y_{i}(t), z_{i}(t)\right)$ at time $t+T_{c}$, or at the beginning of the next communication cycle, where the estimate is obtained via an interpolation of a predetermined trajectory that passes through the waypoints. Using the estimated position, the distance between agents $A_{i}$ and $A_{j}$ at time $t+T_{c}$, denoted $\Delta_{i / j}\left(t+T_{c}\right)$, is computed as

$$
\Delta_{i / j}\left(t+T_{c}\right)=\left\|\mathbf{p}_{i}\left(t+T_{c}\right)-\mathbf{p}_{j}\left(t+T_{c}\right)\right\|_{2}
$$

where $\|\cdot\|_{2}$ is the standard Euclidean norm.

Consider now a configuration of the network where the connectivity is either poor or completely lost. The goal of this research is to study the ability to restore or improve network connectivity via inclusion of an auxiliary agent in the form of an ASV. Because we have no control over the motion of the AUVs, it is impossible to guarantee global connectivity of the network; however, we seek to improve local connectivity at each communication cycle. We do this through a two-stage process that steers the ASV to a waypoint for which the position maximizes the number of connections in the network at a discrete point in time, specifically at the end of the communication cycle. First, a waypoint for the ASV is generated by solving a continuous-time optimal control problem on the duration of the communication cycle $T_{c}$. The objective functional for this optimal control problem is a function of the position of the ASV and the AUVs in the network at the end of the communication cycle. Using the generated waypoint as a target, a second optimal control problem is solved where the objective function is to minimize the time required for the ASV to reach the waypoint. This solution to this second optimal control problem is the control command to the ASV.

\section{Communication Model and Network Connectivity Metrics}

To develop the algorithm described in this paper, we assume a simplified distance-based model for the acoustic modems. Specifically, we use a binary connectivity model, where the strength of the connectivity signal decays to zero when two vehicles are specified distance $\alpha$ from one another. Although underwater communications can vary as a function of many factors, including water depth and quality, we have found through simulation and in-water tests that this model has proved to be sufficiently good as a starting point in this analysis.

It is possible to represent the network of interest as an undirected dynamic graph $G(x(t))=(A, C(x(t)))$, where $A=\left\{A_{1}, \ldots, A_{n}\right\}$ represents the vertices, or agents, in the network and $C(x(t))=\left\{(i, j) \mid \Delta_{i / j}(x(t))<\alpha\right\}$ represents the connections between the agents. We can then define an $n \times n$ adjacency matrix $\mathcal{K}$ where each entry $\left(k_{i j}\right)$ represents the connection between agents $A_{i}$ and $A_{j}$ such that $k_{i j}=1$ when $\left(A_{i}, A_{j}\right) \in C(x(t))$, and $k_{i j}=0$ otherwise. We note that, since $\Delta_{i / j}(x(t))=\Delta_{j / i}(x(t))$, then $a_{i j}=a_{j i}$ and $\mathcal{K}$ is symmetric. Another important measure of the network connectivity is the matrix of node degrees (or the valency matrix), defined as a diagonal matrix that describes the number of connections of each vertex in the graph:

$$
\mathcal{D}(x(t))=\operatorname{diag}\left(\sum_{j=1}^{n} k_{i j}(x(t))\right)
$$

In terms of either the adjacency or node degree matrix, we define the connectivity number $N_{C}$ as the average node degree of the network at each cycle, which can give insight into improvements of local pockets of connectivity within the global network:

$$
N_{C} \equiv \frac{\operatorname{trace}(\mathcal{D}(x(t)))}{n(n-1)}=\frac{\sum_{i=1}^{n} \sum_{j>i}^{n} K_{i j}}{2 n(n-1)}
$$

A second important measure of network connectivity is the manner in which information is propagated from one vehicle to another indirectly through intermediate vehicles. Specifically, indirect information propagation relates how information acquired by one agent will be made available to other agents through secondary connections. To quantify secondary connections, we define the propagation number $N_{P}$ as

$$
N_{P} \equiv N_{C}+\frac{\sum_{i=1}^{n} \sum_{j>i}^{n} \mathcal{S}_{i j}}{2 n(n-1)}
$$


where $\mathcal{S}$ is a $n \times n$ matrix defined as

$$
\begin{aligned}
& \mathcal{S}_{i k}=\left\{\begin{array}{ll}
1 & \mathcal{K}_{i j}=1 \cup \mathcal{K}_{j k}=1 \\
0 & \text { otherwise }
\end{array}, \quad i=1, \ldots, n,\right. \\
& j=i+1, \ldots, n, \quad k=1, \ldots, n, \quad k \neq i, \quad k \neq j
\end{aligned}
$$

and determines if a secondary connection exists between two agents $A_{i}$ and $A_{k}$ that have no direct connection. We note that a propagation number of one implies a strongly connected network, where no agents are excluded.

\section{Autonomous Surface Vehicle Dynamics, Guidance, and Control}

The ASV, denoted as agent $A_{0}$, is controlled over equally spaced time intervals that follow the communication cycles of duration $T_{c}$ time units. Because the speed of the vehicles as viewed by an observer in an inertial (Newtonian) reference frame is sufficiently small, it is reasonable to model the dynamics by including only the kinematics. Since the ASV is constrained to remain on the surface of the water, the two-dimensional kinematic equations that govern the motion of the ASV are given in Universal Transversal Mercator inertial Cartesian coordinates as

$$
\dot{x}=v \cos (\theta), \quad \dot{y}=v \sin (\theta), \quad \dot{\theta}=u
$$

where $x$ and $y$ are the two components of position, $v$ is the inertial speed, $\theta$ is the heading angle (and defines the orientation of the vehicle relative to east), and $u$ is the heading angle rate. To effectively improve the connectivity of the network, it is assumed in this research that the ASV moves with a larger speed than each of the AUVs and that the position of the ASV is unconstrained. We note this is a realistic assumption, as surface vehicle speeds are generally much higher than underwater vehicles.

Autonomous motion of the ASV is accomplished through a waypoint generator in conjunction with a guidance algorithm and an onboard controller. The waypoint is obtained by solving a continuous-time optimal control problem maximizing the number of direct connections available to the ASV. Next, the guidance command used to steer the ASV is obtained by solving a second optimal control problem where it is desired to minimize the time required to attain the waypoint obtained by the waypoint generator. The guidance command is generated every $T_{g}$ time units, where $T_{g}$ is the guidance cycle and $T_{g}<T_{c}$. The onboard controller then attempts to achieve the guidance command. Effectively, the aforementioned process first provides a target for the ASV, and then it controls the vehicle to attain the target as quickly as possible.

\section{A. Optimal Waypoint Generator}

Using the estimated position of the underwater vehicles at time $t+T_{c}$, the next waypoint for the ASV is obtained by solving the following continuous-time optimal control problem. Maximize the cost functional

$$
J=\sum_{i=1}^{n} W_{i} \arctan \left(\alpha-\Delta_{0 / i}\left(t_{0}+T_{c}\right)\right)
$$

subject to the dynamic constraints of Eq. (5), the initial conditions

$$
x\left(t_{0}\right)=x_{0}, \quad y\left(t_{0}\right)=y_{0}, \quad \theta\left(t_{0}\right)=\theta_{0}
$$

the control inequality constraint

$$
|u| \leq u_{\max }
$$

and the terminal inequality time constraint

$$
t_{0}+T_{c} \leq \lambda
$$

where $t_{0}$ is the start time of the communication cycle and $\Delta_{0 / i}\left(t+T_{c}\right)(i=1, \ldots, n)$ is the predicted distance between the ASV and each of the agents $A_{i}(i=1, \ldots, n)$ at the beginning of the next communication cycle. Furthermore, the weights $W_{i}(i=1, \ldots, n)$ in the cost functional of Eq. () are defined as

$$
W_{i}=\frac{t-t_{\text {last }}^{i}}{\lambda}, \quad(i=1, \ldots, n)
$$

where $t_{\text {last }}^{i}$ is the time at which agent $A_{i}$ last transmitted its status message. It is important to note that the cost functional of Eq. (6) is a measure of the number of direct connections between agents and the ASV, thus making it an appropriate measure of the improvement in the connectivity of the network at the end of the next communication cycle. Furthermore, the weights $W_{i}(i=1, \ldots, n)$ in the cost functional of Eq. (6) are chosen so that the ASV moves closer to the vehicles that have been out of communication range for the longest duration. Consequently, Eq. (6) effectively maximizes the number of AUVs within communication range of the ASV with a bias toward those agents that have been excluded from the network. Finally, it is noted that the differential equation $\dot{\theta}=u$ is included in order to constrain the maximum and minimum allowable turn rate on the vehicle, and together with the final time constraint of Eq. (9), ensures that the ASV can reach its optimal point before the end of the guidance cycle, thus providing a more realistic waypoint.

To solve the optimal control problem given in Eqs. (6-9), the necessary information from the status message of each AUV must be supplied to the waypoint generator. Specifically, it is necessary for the ASV to know the location of each AUV at the beginning of the next communication cycle in order to position itself optimally for the beginning of the next set of underwater transmissions. Also important in the status message is the current plan that the network is performing, which indicates how many agents are on task, and the current task each vehicle is performing, which indicates what zone that particular vehicle is patrolling.

Because the optimal control problem of Eqs. (6-9) does not have an analytic solution, it is solved numerically. In this research, we choose to solve the optimal control problem of Eqs. (6-9) using the open-source pseudospectral optimal control software GPOPS [13] together with the nonlinear programming solver SNOPT $[\underline{14}, \underline{15}]$. To obtain a solution to the optimal control problem of Eqs. (믐) as efficiently as possible while still obtaining an accurate solution, 10 Legendre-Gauss collocation points are chosen. Although choosing such a small number of collocation 
may seem to be insufficient, GPOPS uses Legendre-Gauss quadrature approximation, thus making it possible to obtain an accurate waypoint in a computationally efficient manner using a small number of collocation points. While it is beyond the scope of this paper to provide a detailed explanation of pseudospectral methods and their accuracy, details about various pseudospectral methods for solving optimal control problems can be found in [16-20].

As an example of the solution obtained from GPOPS to the optimal control problem of Eqs. (믐), consider the case $n=6$ (that is, five AUVs plus the ASV), $\lambda=150 \mathrm{~s}$, and $\alpha=750 \mathrm{~m}$. In this example, shown in Fig. $\underline{1}$, the AUVs were placed in positions that made it impossible for all AUVs to be within communication range of the ASV. The initial position of the ASV is denoted by a $\diamond$, and its overall path during the time interval is shown as the blue line with triangle markers. Each agent is denoted with a $\bigcirc$ if it is within communication range of the ASV range at the end of the guidance cycle and is denoted with a $\square$ if it is outside communication range of the ASV at the end of the guidance cycle. Figure 1 shows the test case results. First, it is seen for the case where all weights are unity that the ASV attempts to position itself in the middle of the five underwater agents, thus ensuring communication with a maximum possible number of underwater agents. Next, changing the weight of agent $A_{1}$ to $W_{1}=2$ results in the ASV moving closer to $A_{1}$ while still keeping the maximum possible number of AUVs in its communication range. Finally, the weight of agent $A_{2}$ was changed to $W_{2}=3$, where it is seen that the ASV moves to maintain the network connectivity of the three vehicles with the greatest weight.

Finally, as already mentioned, each AUV patrols a particular zone of the area of interest. If a vehicle is outside communication range of the ASV for more than three communication cycles, and thus the ASV has not received its status message, it becomes a priority and a decision is then made to override the optimal waypoint obtained through the waypoint generator and instead go to the center of the zone of that vehicle in an attempt to reestablish a link with that vehicle. This ASV behavior is denoted as polling and is important in underwater applications in order to ensure an underwater vehicle has not drifted outside of the operation area.

\section{B. Guidance Algorithm and Onboard Controller}

The guidance algorithm is in charge of receiving navigation information from the ASV as well as sending steering commands to the onboard controller. The onboard controller runs at the same rate as the guidance cycle, receiving the guidance command and relaying back the current measured position, heading, and speed of the ASV. The guidance command used to steer the ASV consists of heading and speed. The commanded

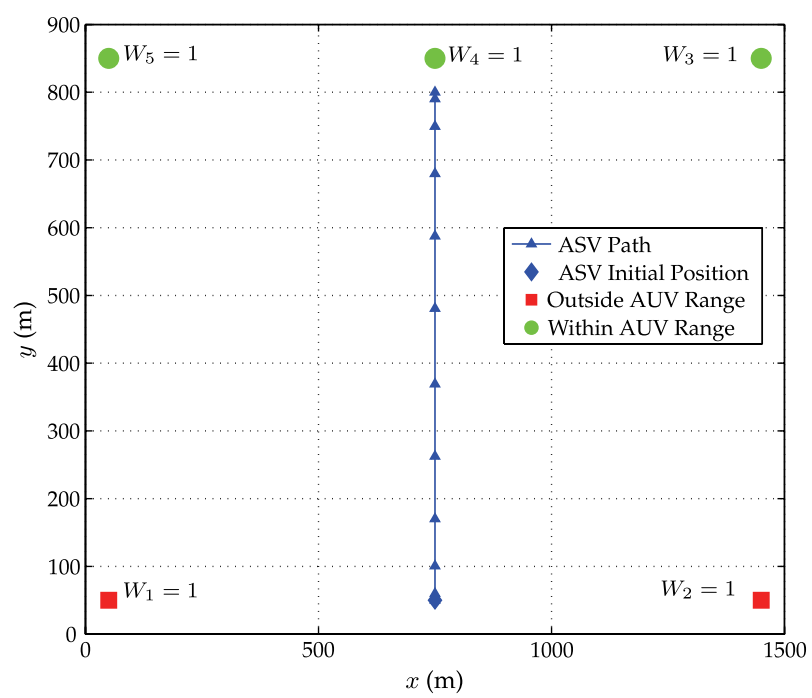

a) Test case 1

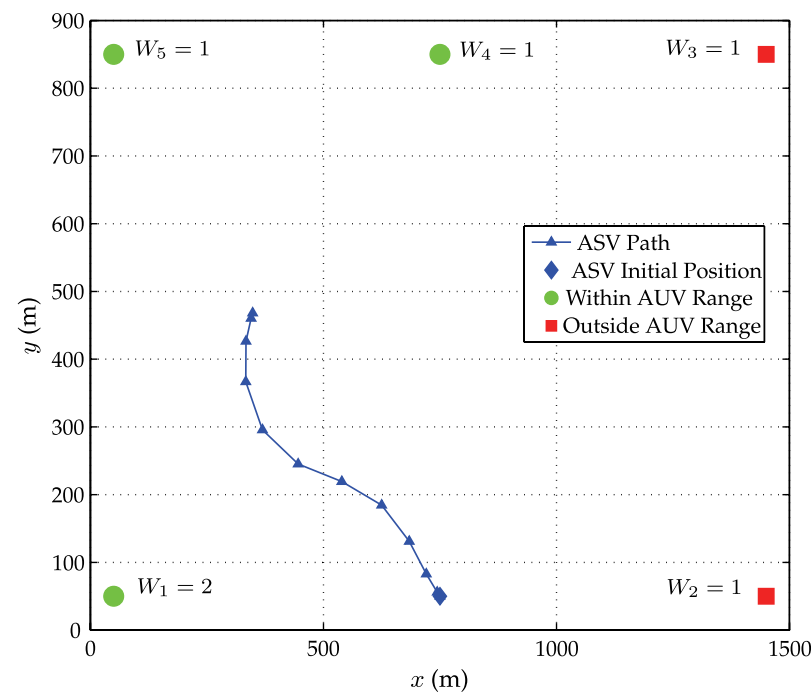

b) Test case 2

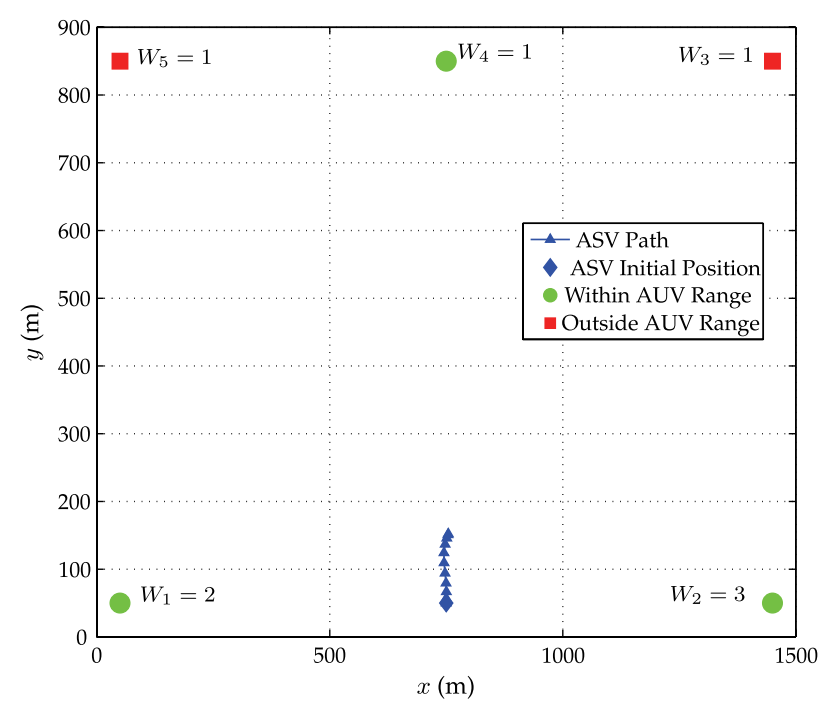

c) Test case 3

Fig. 1 Simulations showing the motion of ASV that optimizes the cost function of Eq. (6). 


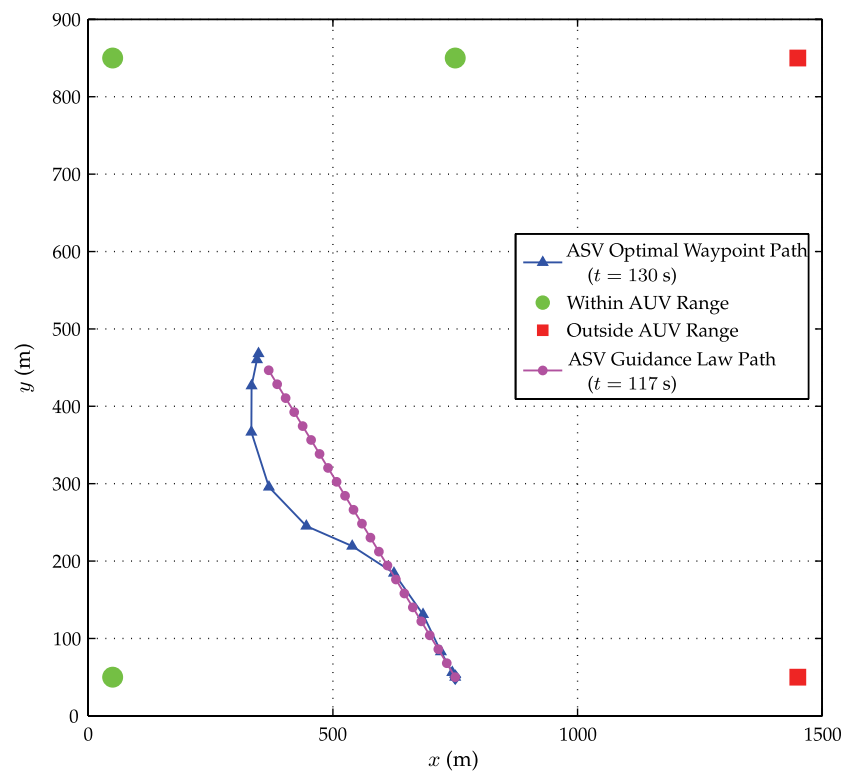

Fig. 2 ASV path obtained using heading angle from the optimal waypoint generator alongside the path obtained using the minimum-time heading angle together with the vehicle rate constraints.

speed of the ASV is constant and preset by a configuration file. The commanded heading is found by solving the following optimal control problem. Minimize

$$
J=t_{f}-t_{0}
$$

subject to the dynamic constraints

$$
\dot{x}=v \cos \theta, \quad \dot{y}=v \sin \theta
$$

and the boundary conditions

$$
x\left(t_{0}\right)=x_{0}, \quad y\left(t_{0}\right)=y_{0}, \quad x\left(t_{f}\right)=x_{f}^{*}, \quad y\left(t_{f}\right)=y_{f}^{*}
$$

where $\left(x_{0}, y_{0}\right)$ is the current position of the ASV, and $\left(x_{f}^{*}, y_{f}^{*}\right)$ is the position of the waypoint generated by the optimal waypoint generator described in Sec. IV.A. It can be shown (see Sec. 2.7 of [21]) that the solution to the optimal control problem of Eqs. (11-13) is given as

$$
\theta^{*}=\tan ^{-1}\left(y_{f}-y_{0}, x_{f}-x_{0}\right)
$$

where the function $\tan ^{-1}(\cdot, \cdot)$ is the four-quadrant inverse tangent. It is important to observe that the rate constraint on the angle $\theta$ has been omitted from the optimal control problem of Eqs. $(\underline{11-13})$ because including the rate constraint on $\theta$ leads to an optimal control problem that does not have an analytic solution.

Figure 2 is an example of an ASV path obtained by solving the control problem of Eqs. (6-9) alongside the path obtained using the guidance law of Eq. (14). It can be seen that the minimum-time solution provides a more direct path to the desired final waypoint.

\section{Separation of Waypoint Generation and Guidance}

Separating the ASV waypoint generation from the ASV guidance has several advantages. First, the waypoint generator numerically solves an optimal control problem that does not have an analytic solution. As a result, this period at which this first optimal control problem is solved (in this case, the duration of a communication cycle $T_{c}$ ) must be much larger than the time required to solve the problem. In this research, it was found that the CPU time required to compute the optimal waypoint was between 1 and $2 \mathrm{~s}$, much less than $T_{c}$. Second, while it is possible to use as the guidance command the heading angle $\theta$ obtained from the solution of the first optimal control problem, this angle is not desirable because the time required to steer the ASV to the optimal waypoint may be very close to the communication cycle. If a sufficiently large disturbance (e.g., a current or wind) alters the predicted path of the ASV, the ASV may not reach the next waypoint within the time constraint. Alternatively, even if the ASV completes the maneuver within the allowable time, it may reach a nonoptimal waypoint, thus reducing the improvement in the connectivity. Second, the minimum-time steering angle (that is, the guidance law) can be solved for analytically, thus making it possible to perform guidance at a much higher rate than that of the communication cycle. Furthermore, the minimum-time steering angle provides a time margin in the sense that the ASV will reach the target sooner than it would have had the optimal waypoint control been used.

\section{Computational Approach}

The aforementioned approach for improving network connectivity was implemented using the Mission Oriented Operating Suite (MOOS)/ Interval Programming (MOOS-IvP) [22] architecture for autonomous control. MOOS-IvP is developed and maintained by the U.S. Naval Undersea Warfare Center (NUWC) in Newport, Rhode Island, the Massachusetts Institute of Technology (MIT), and Oxford University. MOOSIvP is a set of open-source $\mathrm{C}++$ modules for controlling the operations of autonomous marine vehicles. The suite consists of a set of distinct processes communicating through a publish-subscribe database called the MOOSDB. Each variable published to MOOSDB is available to any other processes that have subscribed to that particular variable. Furthermore, variable publications and subscriptions can be performed in real time. 
MOOS was chosen for this research because of its proven ability to handle communication for cooperative control of networks of underwater vehicles [23,24]. MOOS includes the necessary control functions as well as communication modules for simulation and real-time implementation. Moreover, MOOSDB provides the unified interface standard that enables fully autonomous integration of modeling, processing, and control. This allowed for the development of one framework for both simulation and in-water testing. In simulation, the framework could be linked to a vehicle model module, whereas during in-water testing, the framework was incorporated directly into the underwater vehicles.

All AUVs (that is, agents $A_{1}, \ldots, A_{n}$ ) are guided and controlled through modules implemented in MOOS. The control of the AUVs is handled in MOOS via separation between vehicle control and vehicle autonomy. Dynamic control of the AUVs is accomplished via a MOOS process referred to as the front-seat driver, where the front-seat driver provides a navigation and control system steering the vehicle capable of streaming the vehicle's position and trajectory information to the vehicle's autonomy controller, called the backseat driver, and accepting back a stream of autonomy decisions that guide the vehicle such as heading, speed, and depth. Thus, the vehicle is controlled through a frequent exchange of data between the autonomy controller, or the backseat driver, and the dynamic controller, or the front-seat driver; all guidance decisions being made by the autonomy controller, while all navigational information is handled by the dynamic controller [22]. The backseat driver, or the vehicle autonomy, of all underwater agents consists of the MOOS behaviors, which include waypoint, loiter, refuel, and retask. For in-water tests, the front-seat driver controls the vehicle directly [25]. For the purposes of this research, the AUV dynamic model was implemented in C++ and was controlled by input of the desired speed, depth, and heading.

The MOOS communication suite includes processes that parse and distribute the status message of each agent. This stack is exclusively developed and maintained by the MIT Laboratory for Autonomous Marine Sensing Systems [26]. The processes used consist of pAcommsHandler and pGeneralCodec, which are linked through the MOOSDB. The process pAcommsHandler queues all messages that are to be sent by the underwater modem according to a priority level, and pGeneralCodec is a compressor/decompressor that encodes status message data into binary format for transmission by the underwater modem and decodes the data received from the underwater modem. The format of the data is defined in a human-readable extensible markup language (xml) file.

Although the ASV is not guided and controlled by MOOS behaviors, it does use the MOOS communication suite. The ASV waypoint generator and guidance algorithm are implemented in MATLAB ${ }^{\circledR}$ and use a MOOS process called iMATLAB to connect to the MOOSDB and use the MOOS communication processes. The mission is then simulated in real time by running 1) a computer that acts as the environment, simulating the underwater modem and each agent's dynamics models; 2) in another computer equipped with the MOOS communication suite, running each agent's controller along with any necessary autonomy behaviors and the MOOSDB; and 3) running the ASV autonomy controller (consisting of the waypoint generator and the guidance algorithm) along with the MOOS communication suite in a MATLAB-equipped computer. This simulation structure can be seen with all its components in Fig. $\underline{3}$, where MARV denotes the AUV dynamics simulator, and TDMA denotes the communications (comms) status message queuing behavior.

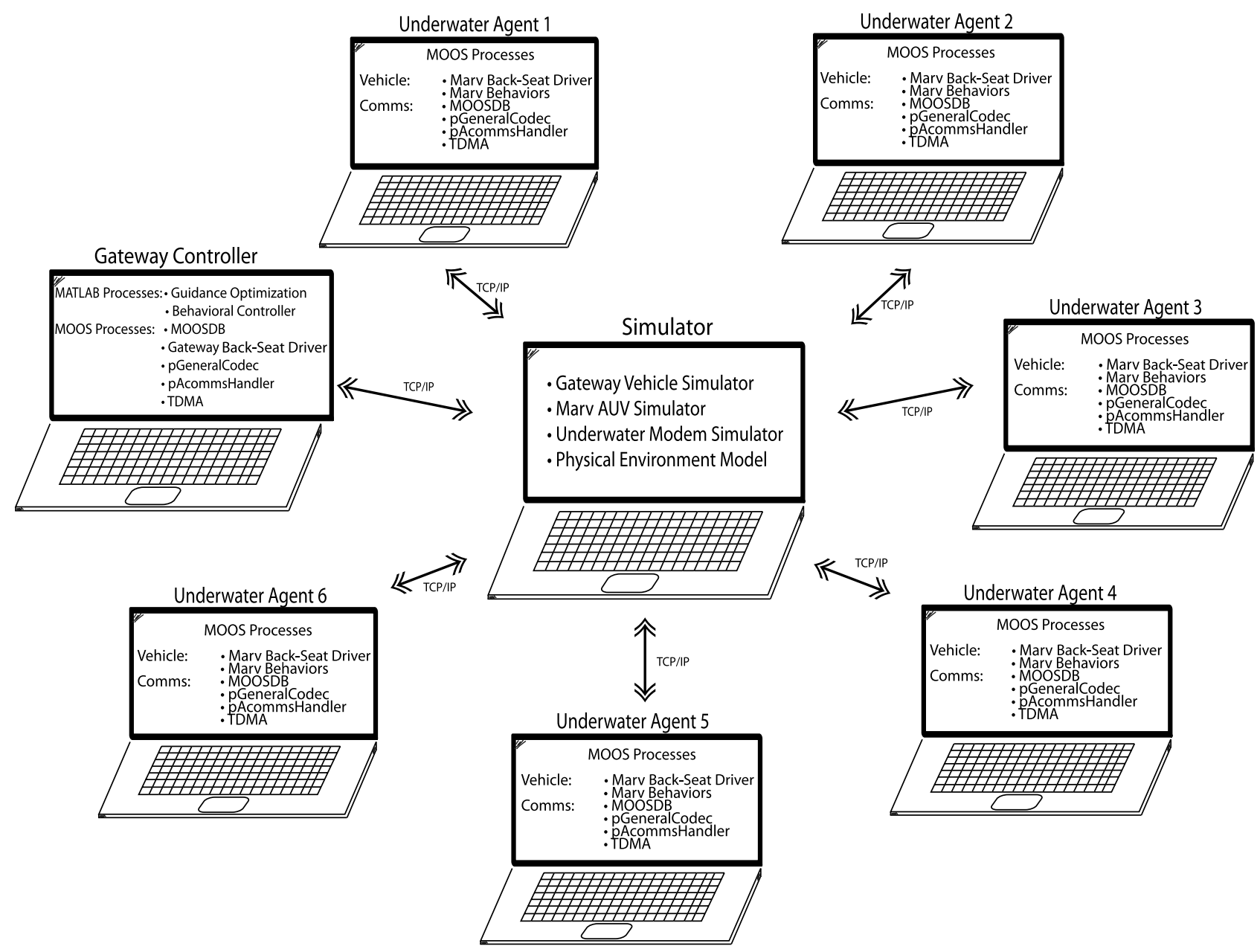

Fig. 3 Schematic of simulation framework. Each computer runs a simulated vehicle along with the necessary MOOS communication processes. 


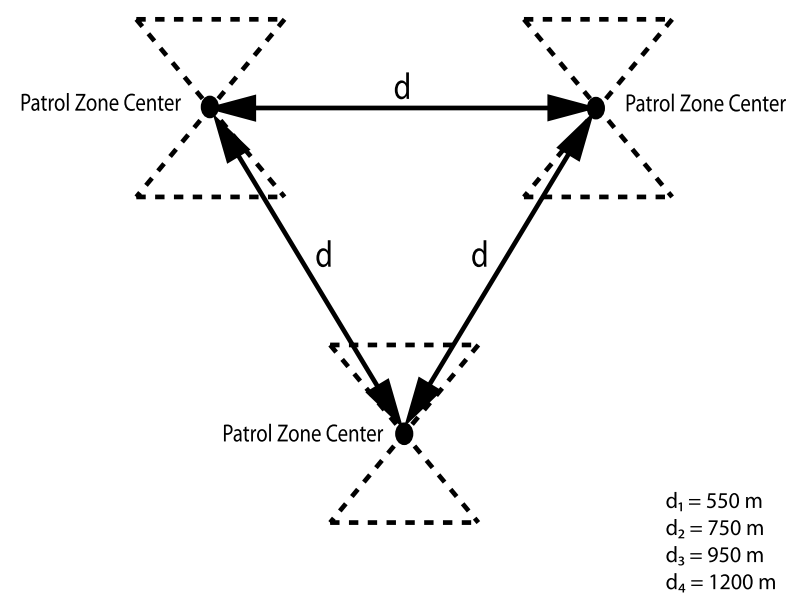

Fig. 4 Mean distances between underwater agents varied in simulation.

\section{Simulation Results}

Simulations were carried out using a maximum of six underwater agents (that is, $n=6$ ). Each simulation is run both without the ASV and with the ASV. To show the performance of the ASV, simulations were performed with varying mean distances $d$ between the AUVs as shown in Fig. 4 . Two particular simulations, called close and spread configurations, are shown in more detail to demonstrate the effectiveness of the ASV. In the close configuration, $d=550 \mathrm{~m}<\alpha$, where $\alpha$ is the communications range parameter; and the AUVs are within communication range of one another. In the spread configuration, $d=750 \mathrm{~m}=\alpha$ and communication between adjacent vehicles was not always possible. Thus, the main role of the ASV in the close configuration is to improve the rate of information propagation in the network. On the other hand, in the spread configuration, the main role of the ASV is to attempt to maintain connectivity in the network, linking as many adjacent agents as possible. Finally, in order to test the polling behavior of the ASV, in the close configuration simulation, a scenario is chosen where one of the vehicles ceased to communicate with the network for an extended period of time (specifically, a duration of six communication cycles).

The connectivity and propagation numbers, $N_{C}$ and $N_{P}$, are shown in Fig. 5 for the close configuration simulation. It is seen that $N_{C}$ and $N_{P}$ increase by including the ASV. The metrics, however, show a decrease in connectivity at the 17th communication cycle. This reduction in connectivity is due to the fact that the extended period of silence from one underwater vehicle in the network leads to the ASV making the decision to attempt to regain communication with the lost vehicle as opposed to maintaining connectivity with the rest of the network. Thus, we see a tradeoff between overall network connectivity and vehicle polling.

Figure 6 shows the connectivity and propagation numbers, $N_{C}$ and $N_{P}$, for the spread configuration simulation. In this case, it is seen that $N_{C}$ and $N_{P}$ are greatly improved with the inclusion of an active ASV. Specifically, it is seen at the beginning of the simulation that the connectivity in the network is not improved because several communication cycles are required before the ASV is in position to assist with improving network connectivity. Once the ASV is in position, however, its inclusion in the network is seen to significantly improve network performance. Finally, Table 1 shows the results of both the close and spread configurations, where it is seen that the ASV improves the connectivity of the network in simulations.

The close and spread configurations demonstrate that including the ASV is most useful when the mean distance between the AUVs is large or when the AUV network is dynamically reconfiguring itself. In either of these cases, the AUV network requires assistance in order to maintain connectivity. The results obtained in the case where $d$ is small are promising, although the addition of the ASV may be redundant because the AUV network is already well connected. To better illustrate the improvement in network connectivity, Fig. 7 shows $N_{C}$ and $N_{P}$ as a function of $d$. These results show a large improvement in connectivity with the addition of the ASV at the upper limit of the modem communication range (that is, $d=750 \mathrm{~m}$ ), thus making the ASV an important asset to the network.

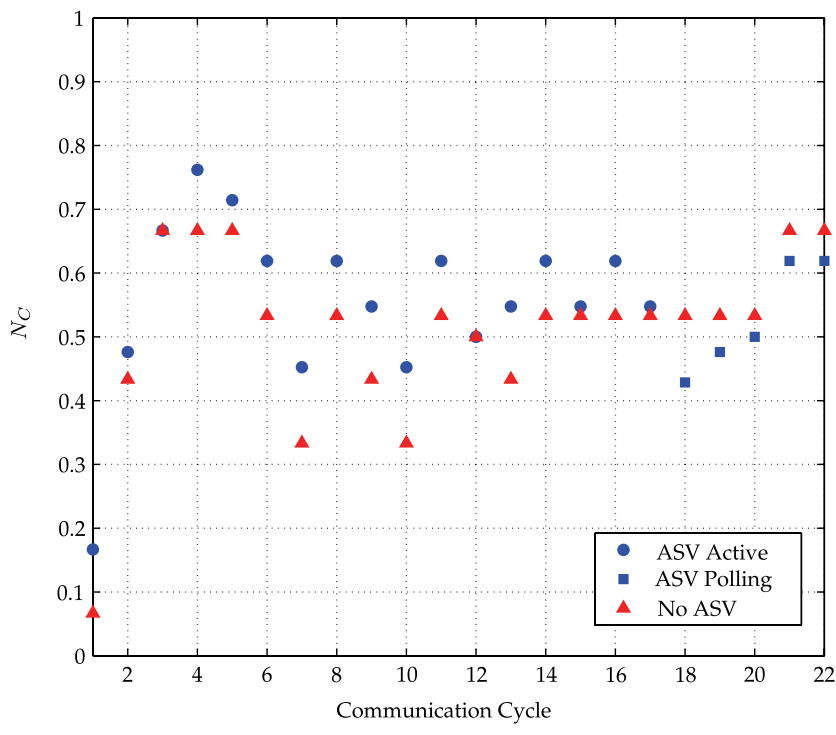

a) Connectivity number $N_{C}$ for close configuration

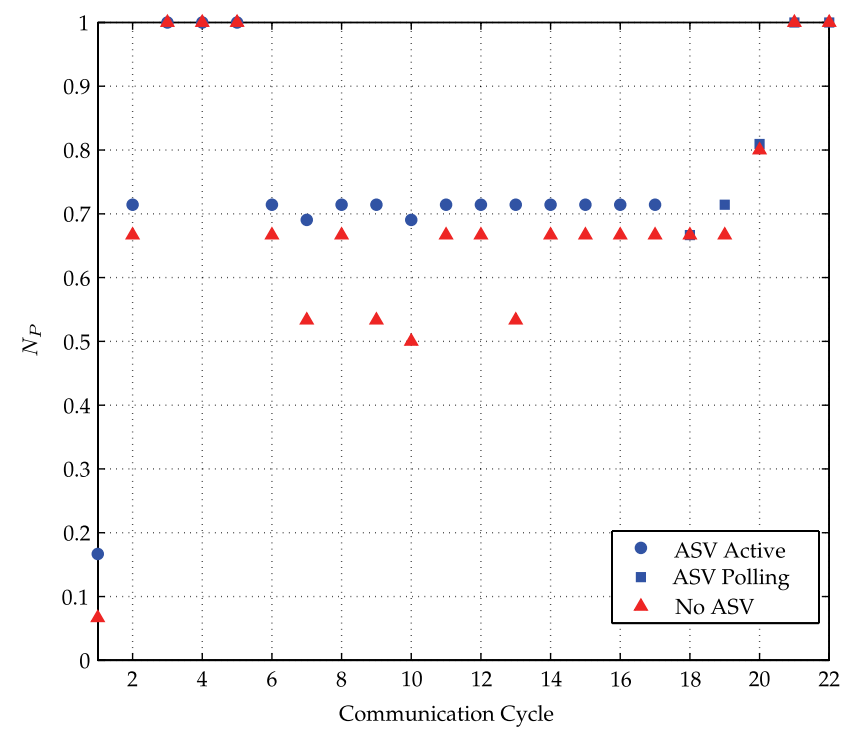

b) Propagation number $N_{P}$ for close configuration

Fig. 5 Connectivity number $N_{C}$ and propagation number $N_{P}$ for the close configuration simulation. 


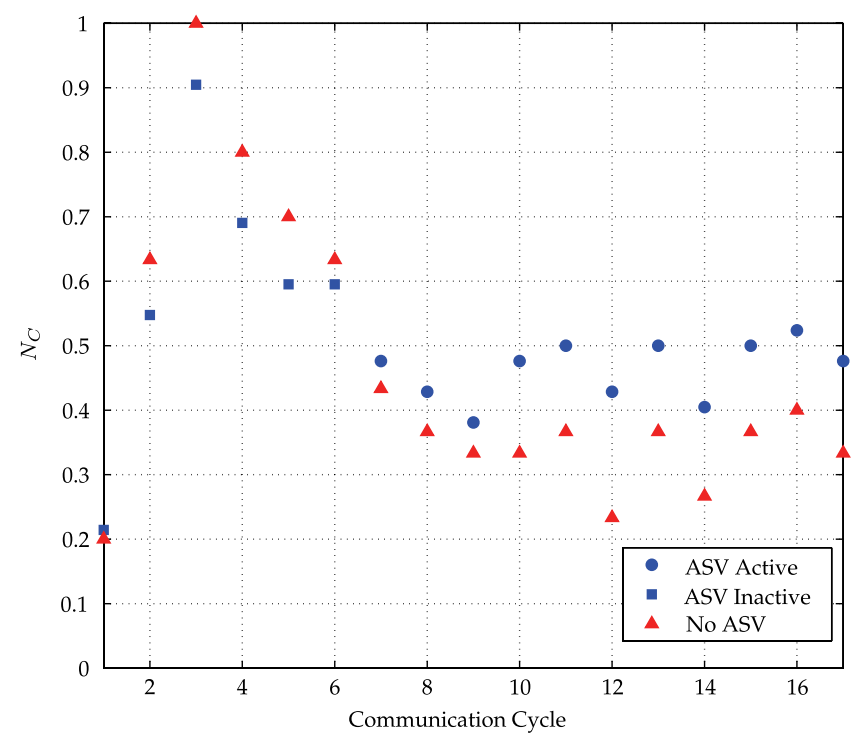

a) Connectivity number $N_{C}$ for spread configuration

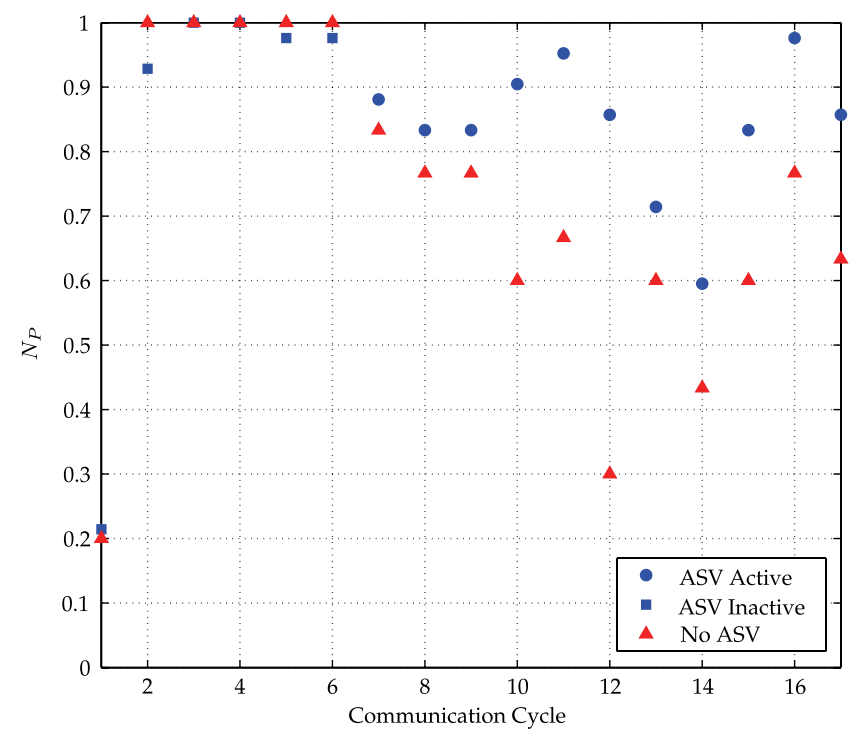

b) Propagation number $N_{P}$ for spread configuration

Fig. 6 Connectivity number $N_{C}$ and propagation number $N_{P}$ for the spread configuration simulation.

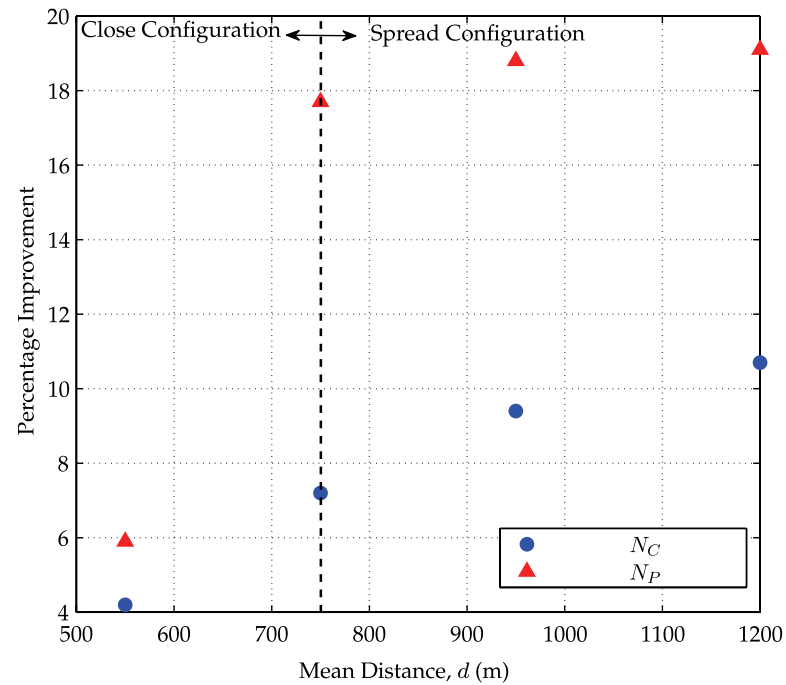

Fig. 7 Improvements in $N_{C}$ and $N_{P}$ as a function of mean distance $d$ from the centers of the AUV patrol zones.

Figure $\underline{8}$ shows the path of the ASV in the close and spread configurations. In the spread configuration, it can be seen that the ASV moves throughout the network in order to propagate information and include as many AUVs as possible. As agents stay out of communication range for longer than three communication cycles, the ASV attempts to return the noncommunicating AUV to the network. In the close configuration, however, the ASV maintains a central position in order to propagate information more efficiently.

\section{In-Water Experiments}

The scenario described in the problem formulation section was implemented and tested at the Naval Undersea Warfare Center's north test range in Newport, Rhode Island. The test was run using a three-underwater-vehicle configuration along with one surface vehicle. The vehicles were arranged in a close configuration, with the center of their patrol set to the coordinates measured in meters from a local origin, as seen in Table 2 .

Three of the main functions of the ASV were tested: 1) the ability of the ASV to correctly place itself in a central communication location relative to the AUVs when initialized in a nonoptimal location, 2) the active repositioning of all AUVs and of the ASV when one underwater vehicle must leave its patrol area in order to refuel, and 3) the polling behavior of the ASV when an underwater vehicle is disconnected from the network for an extended amount of time.

Table 1 Connectivity and propagation numbers for close and spread configurations with and without the ASV

\begin{tabular}{lcccccc}
\hline \hline & \multicolumn{3}{c}{$N_{C}$} & \multicolumn{2}{c}{$N_{P}$} \\
\cline { 2 - 7 } & Without ASV & With ASV & Percentage improvement & Without ASV & With ASV & Percentage improvement \\
\hline Close & 50.9 & 55.1 & 4.2 & 69.5 & 75.4 & 5.9 \\
Spread & 45.6 & 50.8 & 5.2 & 71.5 & 84.3 & 12.7 \\
\hline \hline
\end{tabular}




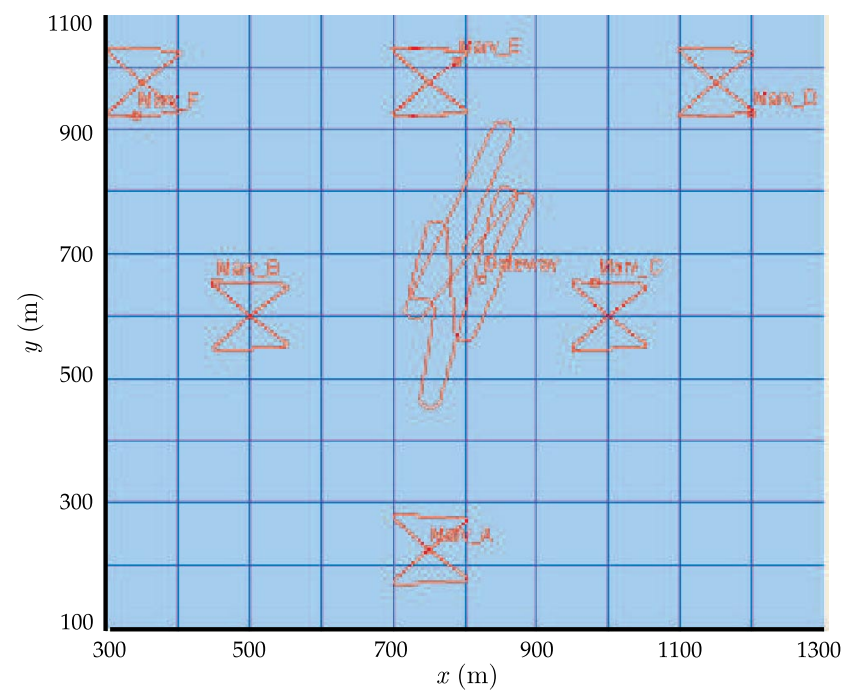

a) Close configuration

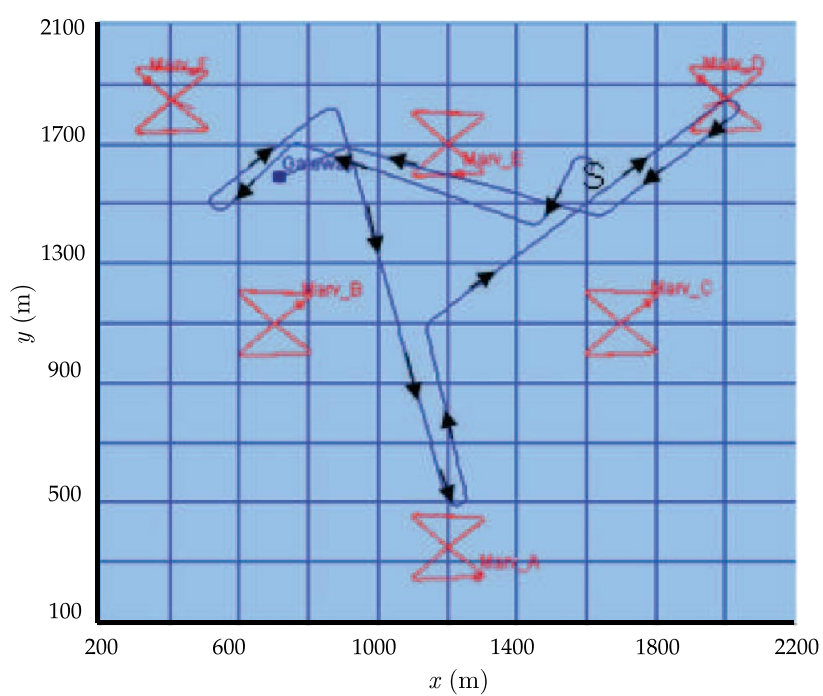

b) Spread configuration

Fig. 8 Snapshots of path taken by the ASV in the close and spread configurations.

\section{A. In-Water Hardware}

In this section, we describe pertinent details of the hardware used for the in-water test conducted at the NUWC north test range. Specifically, this section describes the AUVs, the ASV, and the underwater modem.

\section{Autonomous Underwater Vehicles}

The AUVs used for the in-water testing were Iver2 vehicles provided by the Maritime Autonomy Group at the NUWC. The Iver2 AUV, pictured in Fig. 9, is a small man-portable AUV manufactured in Fall River, Massachusetts, by Ocean Server Technology, Inc. The Iver2 vehicles are approximately $65 \mathrm{in}$. in length and weigh approximately $50 \mathrm{lb}$. These vehicles are $5 \mathrm{in}$. longer than the standard Iver vehicles and were lengthened to provide space for additional electronics, including a precision clock, an inertial measurement unit (IMU), and a iridium satellite transceiver. The vehicles can operate at speeds of up to $4 \mathrm{kt}$ using rechargeable batteries that have $600 \mathrm{~W} \cdot \mathrm{h}$ of capacity. Vehicle endurance is anywhere between 4 and $12 \mathrm{~h}$, depending on the vehicles speed and hotel load (e.g., A/D conversion for the acoustic array). The vehicles also come equipped with integrated depth (pressure) and altitude sensors. The Iver2 AUV is also equipped with the YSI 6600 V2 sonde Woods Hole Oceanographic Institute (WHOI) acoustic micromodem. The payload computer stack consists of a PIII-800 CPU and two eight-channel D/A boards.

Each of the Iver2 vehicles is equipped with a Global Positioning System (GPS) receiver and a three-axis digital compass providing roll, pitch, and yaw. One of the vehicles was recently equipped with a Doppler velocity logger to provide closed-loop speed control. The other two vehicles have open-loop speed control using speed tables based on thruster revolutions per minute. Navigation solutions are computed using dead reckoning and sent to the backseat over the backseat/front-seat interface. The Iver2 vehicles use WiFi communication while on the surface and micromodem communication while underwater. The acoustic modem is used both for providing status messages to the topside as well as for reception of command and control (C2) messages from the topside.

\section{Autonomous Surface Vehicle}

The ASV used for the in-water testing was provided by the NUWC's Maritime Unmanned Systems Facility (MUSF). Each ASV comes with three distinct assets required for operation: 1) an ASV platform, 2) a host station housed in a storage container express (CONEX), and 3) a C2 workstation. Figure 10 shows all three of these components. The platform used during testing was the NUWC-4, the latest ASV developed by the MUSF. The exterior dimensions of the NUWC-4 vehicle are consistent with the U.S. Navy standard $11 \mathrm{~m}$ rigid-hulled inflatable boat. The dynamics of the ASV, propulsion status, power, and environmental data are monitored remotely from the C 2 workstation. The ASV is equipped with GPS and IMU sensors to support navigation.

The ASV host platform consists of a $15 \mathrm{ft}$ CONEX box with telescoping poles to support the host antenna array system. The CONEX box houses the $\mathrm{C} 2$ workstation and must be located within line-of-sight range of the ASV operating area for transmission of navigational data. The $\mathrm{C} 2$ operator has the capability of loading and initiating the execution of a route, which consists of a series of waypoints to the ASV core system. The $\mathrm{C} 2$ operator also has the capability to send throttle, rudder, and transmission control commands to the ASV core system in order to perform emergency maneuvers as required. For this in-water experiment, an onboard computer running the ASV algorithm provided all guidance commands, which were then sent to the $\mathrm{C} 2$ operator who executed all commands remotely.

Table 2 Position of the center of the patrol zone for each AUV in the in-water test

\begin{tabular}{lcc}
\hline \hline & \multicolumn{2}{c}{ Origin } \\
\cline { 2 - 3 } & Latitude $=41: 540000 \mathrm{deg}$ & Latitude $=-71: 349167 \mathrm{deg}$ \\
\hline Agent & $x, \mathrm{~m}$ & $y, \mathrm{~m}$ \\
\hline $\mathrm{A}$ & 450 & 150 \\
$\mathrm{~B}$ & 150 & 700 \\
$\mathrm{C}$ & 750 & 700 \\
\hline \hline
\end{tabular}




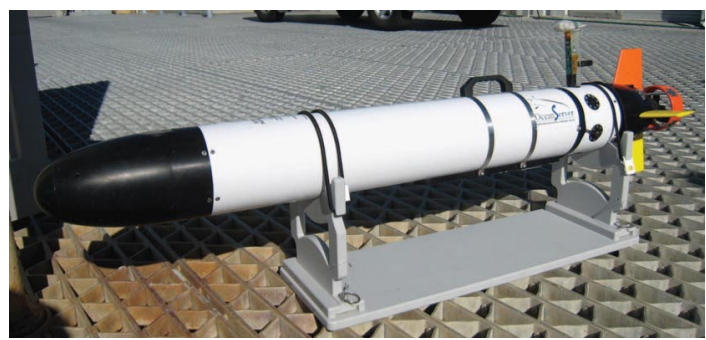

Fig. 9 Iver2 AUV used for the in-water testing.
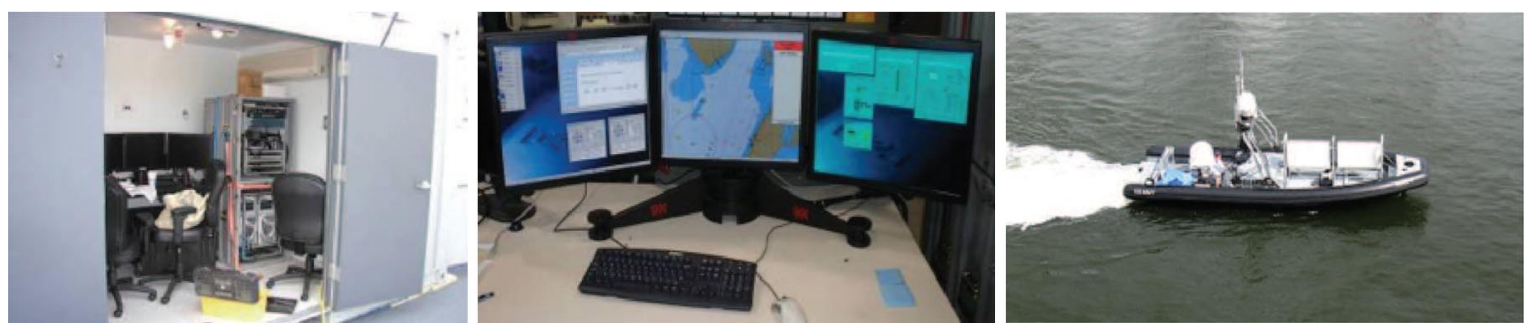

Fig. 10 ASV used for the in-water testing. Each ASV comes with a host station contained within a storage CONEX (left), a C2 workstation (middle), and an ASV platform (right).

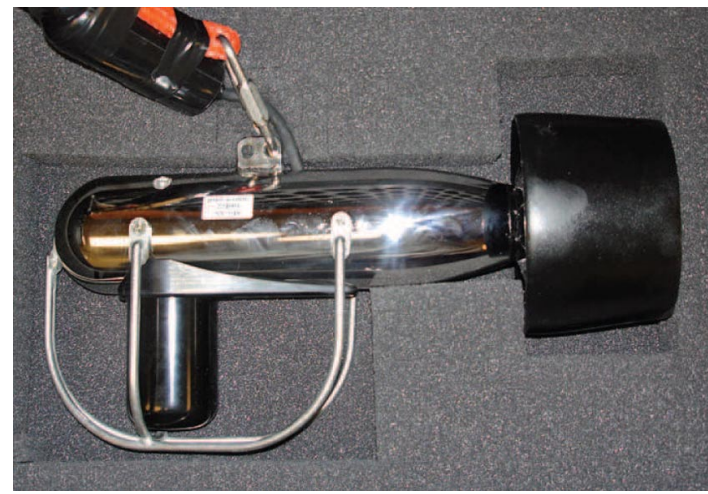

Fig. 11 Underwater acoustic modem used for communication and status message relaying among agents during the in-water testing.

\section{Underwater Modem}

The underwater acoustic modem used for communication and status message relaying among agents during the in-water testing was designed by the Acoustic Communications Group at the WHOI. The WHOI micromodem is a compact low-power underwater acoustic communication and navigation subsystem. It has the capability to perform low-rate frequency-hopping frequency-shift keying, variable rate phase-coherent keying, and two different types of long baseline navigation: narrowband and broadband [27]. For the purposes of this in-water test, the modem was configured to send and receive a $32 \mathrm{~B}$ message and the default data rate was $80 \mathrm{bps}$. The system operates at approximately 10,15 , or $25 \mathrm{kHz}$ and uses $4 \mathrm{kHz}$ of bandwidth. To allow for the ASV to communicate with the underwater vehicles, a transducer towfish, shown in Fig. 11 , was towed by the ASV.

\section{B. In-Water Results}

The connectivity and propagation numbers for the in-water tests were calculated from the log files each agent collected during the tests. The three ASV functions tested were divided into two main experiments, following the same format as in simulation. In the first experiment, the ASV was initialized in a position on the corner of the operation area, and as it acquired information about the underwater network, it correctly positioned itself in an optimal position to propagate information among underwater agents. In the second experiment, the ASV was initialized in a central location relative to the underwater agents, and after a number of intervals, one of the underwater agents ceased communication such that the ASV's polling behavior was tested.

Figure 12 shows the results for the first in-water test. We note that the connectivity and propagation numbers are worse for the network with the ASV included while the ASV is traveling to optimally position itself, as was seen in simulation. Once the ASV is correctly positioned, the improvements in connectivity and propagation numbers are obvious with the inclusion of the ASV in the network. Figure 13 shows the results for the second in-water test. It can be seen that the connectivity and propagation numbers are increased with the addition of the ASV. The connectivity, however, decreases at communication cycle 13 due to the fact that one of the AUVs ceases to communicate with the network for an extended period of time. As a result, the ASV moves to attempt to restore communication with the noncommunicating vehicle. This last result shows that the ASV will trade overall network connectivity in order to attempt to regain communication with a lost vehicle.

Table 3 summarizes the mean improvements in connectivity made with the addition of the ASV to the underwater network for each in-water test. From these results, it can be seen that the improvements for the in-water tests were higher than in simulation. Simulation results assumed perfect communication, whereas actual in-water communication have a number of different factors that can hinder a message from reaching its target. This result shows that the ASV concept is most useful when used in situations of intermittent and uncertain communication. 


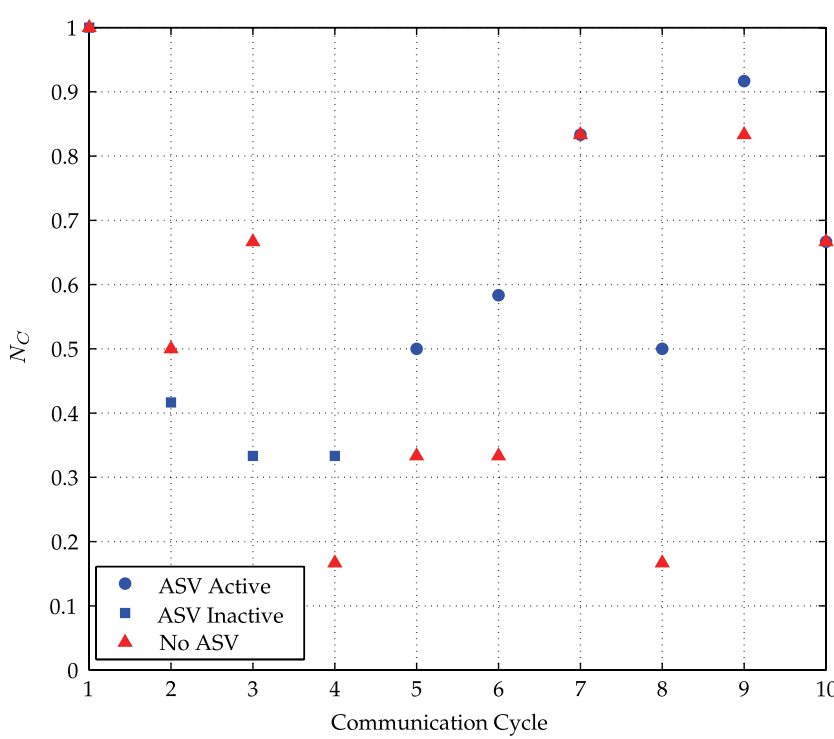

a) Connectivity number $N_{C}$ for first in-water test

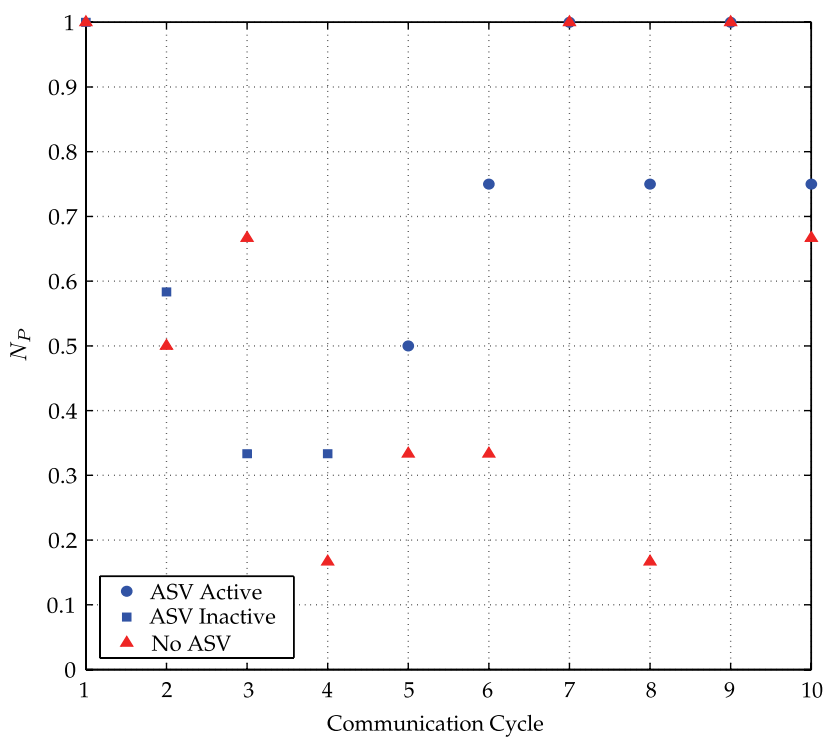

b) Propagation number $N_{P}$ for first in-water test

Fig. 12 Connectivity number $N_{C}$ and propagation number $N_{P}$ for the first in-water test.

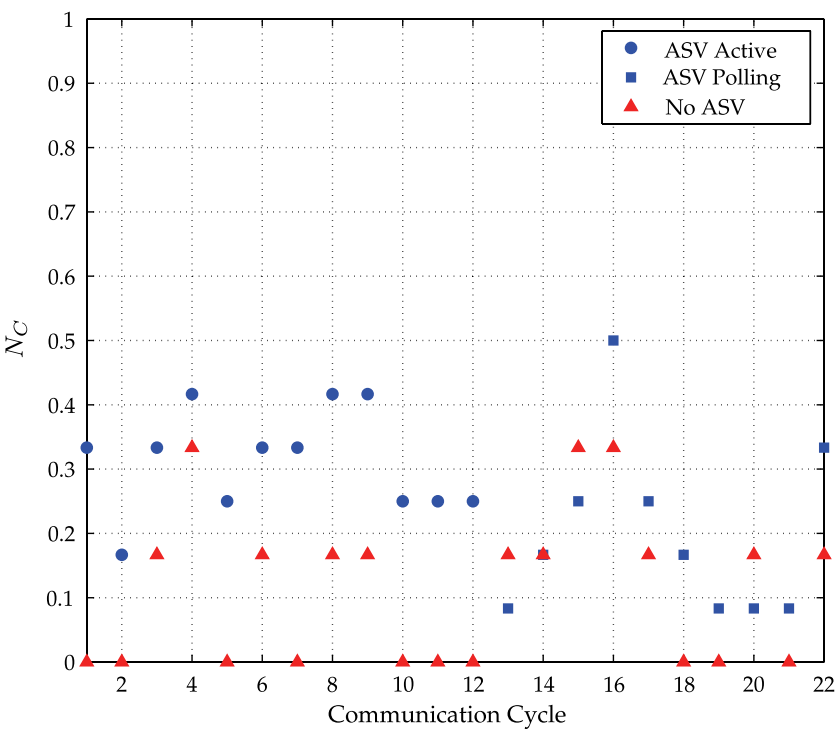

a) Connectivity number $N_{C}$ for second in-water test

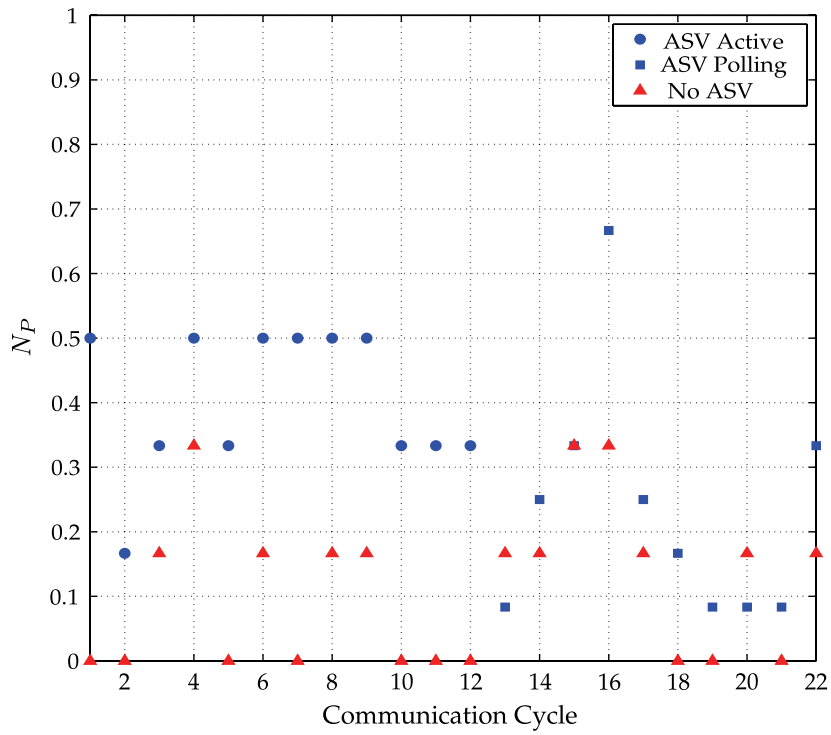

b) Propagation number $N_{P}$ for second in-water test

Fig. 13 Connectivity number $N_{C}$ and propagation number $N_{P}$ for the second in-water test.

\section{Discussion}

We have seen that the addition of an ASV can improve the connectivity of a network of AUVs while still leaving each vehicle free to perform its mission task. The simulations performed in this research show average improvements of 5 and $12 \%$, respectively, in connectivity number and propagation number throughout the entire simulation. Note, however, that these average numbers can be skewed due to the initial positioning and initialization of the ASV. Actual improvement of up to 20 and 55\% were seen in the connectivity and propagation numbers, respectively, for particular communication cycles. The results given here indicate that including an ASV is most beneficial in a dynamically reconfigurable network of underwater vehicles, such that it can ensure that no vehicles will be left out of connectivity for extended periods of time. We have also seen that the ASV is useful when used in situations of intermittent and uncertain communication, such as what is often seen in real-world underwater applications. In a case where the network is already connected on a known mission path, the use of an ASV may be costly and redundant, as an extra vehicle must be added to the network for its implementation. In situations, however, where network connectivity is

Table 3 Connectivity and propagation numbers for in-water tests ${ }^{\mathrm{a}}$

\begin{tabular}{lcccccc}
\hline \hline & \multicolumn{3}{c}{$N_{C}$} & \multicolumn{2}{c}{$N_{P}$} \\
\cline { 2 - 7 } & Without ASV & With ASV & Percentage improvement & Without ASV & With ASV & Percentage improvement \\
\hline Test 1 & 55.0 & 60.8 & 5.8 & 58.3 & 70.0 & 11.7 \\
Test 2 & 11.4 & 26.2 & 14.8 & 11.4 & 32.6 & 21.2 \\
\hline \hline
\end{tabular}

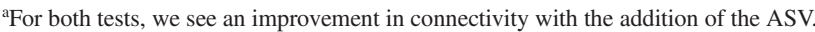


unreliable or even nonexistent, the increase in connectivity provided by the ASV may outweigh the cost of adding an extra vehicle and allow a network to perform a mission that may otherwise not be possible to accomplish.

\section{Conclusions}

A new approach for improving the connectivity of an underwater vehicle network by introducing an ASV into the network has been studied. An algorithm has been developed that consists of an optimal waypoint generator and a minimum-time guidance law. The approach is designed to improve the number of connections in the network, thus improving the overall connectivity of the network. Simulations were performed for different network configurations, and it was found that the inclusion of the ASV improves network communication. In-water experiments were also run using a network of three underwater vehicles and one surface vehicle. Results of the in-water experiments were consistent with the simulations, demonstrating that the inclusion of the ASV improves network connectivity.

\section{Acknowledgments}

The authors gratefully acknowledge support for this research from the U.S. Office of Naval Research under grant N00014-08-1-1173 and contract N0001410WX20839. The authors would also like to thank Scott Sideleau, Donald Eickstedt, Gary Choplin, and Kyle DeMedeiros for their help and support during the course of this research.

\section{References}

[1] Zavlanos, M. M., and Pappas, G. J., "Distributed Connectivity Control of Mobile Networks," IEEE Transactions on Robotics and Automation, Vol. 24, No. 6, Dec. 2008, pp. 1416-1428. doi:10.1109/TRO.2008.2006233

[2] Zavlanos, M. M., and Pappas, G. J., "Potential Fields for Maintaining Connectivity of Mobile Networks," IEEE Transactions on Robotics and Automation, Vol. 23, No. 4, Aug. 2007, pp. 812-816. doi:10.1109/TRO.2007.900642

[3] Sweeney, J., Brunette, T., Yang, Y., and Grupen, R., "Coordinated Teams of Reactive Mobile Platforms," Proceedings of the IEEE International Conference on Robotics and Automation, Washington, D.C., IEEE Publ., Piscataway, NJ, 2002, pp. 299-304.

[4] Thibodeau, B., Fagg, A., and Levine, B., Signal Strength Coordination for Cooperative Mapping, Univ. of Massachusetts, Department of Computer Science, Amherst, MA, 2004

[5] Wagner, A., and Arkin, R., "Multi-Robot Communication-Sensitive Reconnaissance," Proceedings of the IEEE International Conference on Robotics and Automation, New Orleans, LA, IEEE Publ., Piscataway, NJ, June 2004, pp. 4674-4681. doi:10.1109/ROBOT.2004.1302455

[6] Hsieh, M. A., Cowley, A., Kumar, V., and Taylor, C., "Maintaining Network Connectivity and Performance in Robot Teams," Journal of Field Robotics, Vol.24, No. 4, Vol. 25, No. 1, Jan. 2008, pp. 111-131. doi:10.1002/rob.20221

[7] Han, Z., Swindlehurst, A. L., and Liu, K. J. R., "Optimization of MANET Connectivity Via Smart Deployment/Movement of Unmanned Air Vehicles,” IEEE Transactions on Vehicular Technology, Vol. 58, No. 7, July 2009, pp. 3533-3546. doi:10.1109/TVT.2009.2015953

[8] Graduate Texts in Mathematics, 3rd ed., Springer-Verlag, New York, 2005.

[9] Cormen, T. H., Leiserson, C. E., Rivest, R. L, and Stein, C., Introduction to Algorithms, MIT Press, Cambridge, MA, 2001.

[10] Flake, G. W., Tarjan, R. E., and Tsioutsiouliklis, K., "Minimum Cut Tree Clustering," Internet Mathematics, Vol. 1, No. 4, 2002 , pp. $385-408$. doi:10.1080/15427951.2004.10129093

[11] Vargo, E. P., Kincaid, R. K., and Alexandrov, N., "Toward Optimal Transport Networks," 12th AIAA/ISSMO Multidisciplinary Analysis and Optimization Conference, AIAA Paper 2008-5814, Sept. 2008.

[12] Fiedler, M., "Algebraic Connectivity of Graphs," Czechoslovak Mathematical Journal, Vol. 23, No. 2, 1973, pp. $298-305$. doi: $10.1080 / 03081080500054810$

[13] Rao, A. V., Benson, D. A., Darby, C. L., Francolin, C., Patterson, M. A., Sanders, I., and Huntington, G. T., "Algorithm 902: GPOPS, A Matlab Software for Solving Multiple-Phase Optimal Control Problems Using the Gauss Pseudospectral Method," ACM Transactions on Mathematical Software, Vol. 37, No. 2, April-June 2010, pp. 22:1-22:39. doi: $10.1145 / 1731022.1731032$

[14] Gill, P. E., Murray, W., Saunders, M. A., and Wright, M. H., User's Guide for NPSOL (Version 4.0): A FORTRAN Package for Nonlinear Programming, Department of Operations Research, Stanford Univ., Palo Alto, CA, Jan. 1986.

[15] Gill, P. E., Murray, W., and Saunders, M. A., "SNOPT: An SQP Algorithm for Large-Scale Constrained Optimization," SIAM Review, Vol. 47, No. 1, 2005, pp. 99-131. doi: $10.1137 / \mathrm{S} 0036144504446096$

[16] Benson, D. A., "A Gauss Pseudospectral Transcription for Optimal Control," Ph.D. Thesis, Massachusetts Inst. of Technology, Cambridge, MA, 2004.

[17] Benson, D. A., Huntington, G. T., Thorvaldsen, T. P., and Rao, A. V., "Direct Trajectory Optimization and Costate Estimation via an Orthogonal Collocation Method," Journal of Guidance, Control, and Dynamics, Vol. 29, No. 6, Nov.-Dec. 2006, pp. 1435-1440. doi: $10.2514 / 1.20478$

[18] Huntington, G. T., "Advancement and Analysis of a Gauss Pseudospectral Transcription for Optimal Control," Ph.D. Thesis, Department of Aeronautics and Astronautics, Massachusetts Inst. of Technology, Cambridge, MA, 2007.

[19] Garg, D., Patterson, M. A., Darby, C. L., Francolin, C., Huntington, G. T., Hager, W. W., and Rao, A. V., "Direct Trajectory Optimization and Costate Estimation of Finite-Horizon and Infinite-Horizon Optimal Control Problems via a Radau Pseudospectral Method," Computational Optimization and Applications, Vol. 49, No. 2, Oct. 2009, pp. 335-358. doi:10.1007/s10589-009-9291-0

[20] Garg, D., Patterson, M. A., Hager, W. W., Rao, A. V., Benson, D. A., and Huntington, G. T., "A Unified Framework for the Numerical Solution of Optimal Control Problems Using Pseudospectral Methods," Automatica, Vol. 46, No. 11, Nov. 2010, pp. 1843-1851. doi:10.1016/j.automatica.2010.06.048

[21] Bryson, A. E., and Ho, Y.-C., Applied Optimal Control, Hemisphere, New York, 1975.

[22] Benjamin, M. R., Newman, P. M., Schmidt, H., and Leonard, J. J., "An Overview of MOOS-IvP and a Brief Users Guide to the IvP Helm Autonomy Software," Massachusetts Inst. of Technology, Dept. of Mechanical Engineering TR MIT-CSAIL-TR-2009-028, Cambridge, MA, June 2009.

[23] Eickstead, D. P., Benjamin, M. R., Wang, D., and Schmidt, H., "Behavior Based Adaptive Control for Autonomous Oceanographic Sampling," Proceedings of the IEEE International Conference on Robotics and Automation, IEEE Publ., Piscataway, NJ, April 2007, pp. 4245-4250.

[24] Eickstead, D. P., Benjamin, M. R., Schmidt, H., and Leonard, J. J., "Adaptive Control of Heterogeneous Marine Sensor Platforms in an Autonomous Sensor Network," Proceedings of the IEEE International Conference on Intelligent Robots and Systems, IEEE Publ., Piscataway, NJ, Oct. 2006, pp. 5514-5521. 
[25] Eickstead, D. P., and Sideleau, S. R., "The Backseat Control Architecture for Autonomous Robotic Vehicles: A Case Study with the Iver2 AUV," Proceedings of IEEE/MTS Oceans'09 Conference, Biloxi, MS, IEEE Publ., Piscataway, NJ, Oct. 2009, pp. 1-8.

[26] Schmidt, H., Balasuriya, A., and Schneider, T., MOOS-IvP Autonomous Command and Control for Undersea Network Nodes User's Guide, Massachusetts Inst. of Technology, Dept. of Mechanical Engineering, Cambridge, MA, Dec. 2008.

[27] Freitag, L., Grund, M., Singh, S., Partan, J., Koski, P., and Ball, K., "The WHOI Micro-Modem: An Acoustic Communications and Navigation System for Multiple Platforms," Proceeding of the IEEE Oceans, Washington, D.C., Vol. 2, IEEE Publ., Piscataway, NJ, Sept. 2005, pp. $1086-1092$. doi: $\underline{10.1109 / \text { OCEANS.2005.1639901 }}$

C. Rouff

Associate Editor 